\title{
NeuroRehab News
}

\section{Caminar Después de una Lesión Medular Completa. ¿Ciencia o Milagro?}

Neurorehabilitación y Trastornos del Movimiento

\section{Resumen:}

Tn estudio recientemente publicado en pacientes con lesión medular ( 7 completa y 1 parcial) sugiere un nuevo paradigma en la rehabilitación de estos pacientes añadiendo como mejora la inclusión de estímulos táctiles. Tras 12 meses de tratamiento combinado, en el que se incluye la realidad virtual inmersiva y la marcha con exoesqueletos controlados mediante la interfaz hombre-ordenador se descubrió que la mitad de los pacientes mejoraron en variables relacionadas con la sensación de estímulos y también en la contracción voluntaria de algunos de los músculos más importantes en la marcha.

Como sabemos, la ciencia avanza a pasos agigantados en ciertos sectores, esta vez ha tocado a las ciencias de la salud, concretamente al área de la neurorrehabilitación en la lesión medular. Y es que hasta hace pocos meses la esperanza de que una persona con una lesión medular completa pudiera volver a sentir estímulos sensitivos o a realizar contracciones de forma voluntaria por debajo del nivel de la lesión era imposible. A raíz de una investigación publicada por Ana Donati en Scientific Reports (de Nature), una de las mejores revistas científicas a nivel mundial, estas esperanzas han cambiado. Esta investigación ha sido llevada a cabo por varios grupos de Brasil y Estados Unidos dirigidos por el Dr. Miguel Nicolelis, pionero en el estudio y codificación de interfaces humano-ordenador, término acuñado en inglés Brain Machine Interface (BMI). A modo de resumen, este sistema que utiliza unos electrodos situados en la cabeza de forma externa mediante un gorro, capta las señales eléctricas de la corteza cerebral para después enviarlas a un ordenador e interpretarlas, los datos obtenidos se pueden utilizar, por ejemplo, para mover un exoesqueleto.

El estudio publicado está basado en una interfaz BMI, pero aportaron algo que el resto de estudios no aportan y es la estimulación táctil durante el protocolo de tratamiento de estos pacientes. El ensayo clínico se llevó a cabo en 8 pacientes, 7 de ellos con una lesión medular completa según los criterios de la Asociación Americana de Lesionados Medulares (en inglés ASIA) y uno parcial, con más de 1 año padeciendo dolor derivado de la lesión. Este protocolo se basó en un tratamiento multidisciplinar en el que los pacientes estuvieron durante 12 meses haciendo de media 5 horas y media diarias de rehabilitación, resumen en la Figura A.

La rehabilitación realizada combinó el entrenamiento intensivo de realidad virtual inmersiva, la realimentación visual-táctil y caminar con dos exoesqueletos facilitados por una interfaz BMI y en uno de ellos incluyendo un sistema diseñado específicamente para la estimulación táctil. Resumen en la Figura B.

Los resultados fueron sorprendentes ya que la mitad de las personas que participaron en el ensayo clínico a los 12 meses mostraron capacidad de sentir estímulos y contraer musculatura que antes no eran capaces de realizar por si solos, pasando de una clasificación de lesión medular completa a una parcial. Las mejoras fueron en musculatura que es relevante a la hora de realizar la marcha humana.

¿Por qué solo mejoraron la mitad? A raíz de un estudio llevado a cabo por Kakulas en el que descubrieron que aproximadamente el 60\% de los pacientes diagnosticados clínicamente como lesión medular completa todavía tenían entre un 2 y un 27\% del área de trasmisión
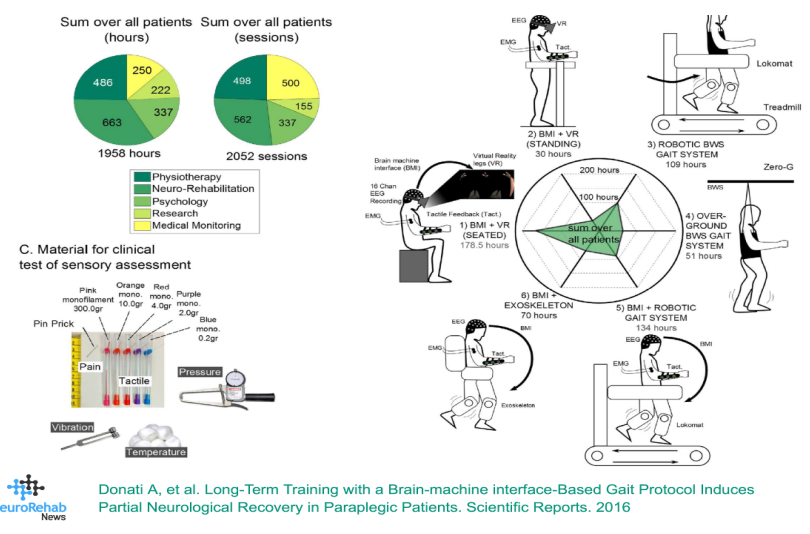

"Añadir estímulos táctiles aparte del estímulo motor al protocolo de rehabilitacion puede proporcionar más información a la médula espinal y favorecer los fen6menos de neuroplasticidad" 
(sustancia blanca) en la médula espinal preservado. Esto, apoyado por otro estudio que dice que este tipo de pacientes, aunque clínicamente se presenten como lesionado medular completo, en más del $80 \%$ de los casos los axones pueden tener funcionalidad.

Lo que lleva a los autores a la hipótesis de que el añadir estímulos táctiles, aparte del estímulo motor, al protocolo de rehabilitación puede proporcionar más información a la médula espinal y se genere un fenómeno de neuroplasticidad con los axones que se presentan funcionales. También se debe tener en cuenta que este es un estudio realizado en personas con la lesión medular durante más de un año. ¿Qué pasaría si este protocolo se aplica a personas con la lesión medular reciente? Esta es otra vía a explorar y que además parece muy prometedora.

La investigadora Donati, tras realizar este estudio plantea la necesidad de mejorar las aplicaciones de BMI añadiendo más estímulos para ayudar a los pacientes a recuperar la movilidad, a través del uso de la prótesis controlada por el cerebro. A primera vista, esta es una terapia potencialmente nueva de neurorrehabilitación, capaz de inducir recuperación parcial de funciones en el paciente con lesión medular.

\section{"Después de 12 meses de re- habilitación la mitad de los pacientes tenían contrac- ción voluntaria"}

\section{Conclusión:}

El tratamiento de rehabilitación puede ser muy beneficioso para los pacientes lesionados medulares cuya lesión no suponga la sección anatómica de la médula espinal. La combinación de nuevas tecnologías y el estímulo táctil al mismo tiempo puede mejorar los resultados de recuperación.

\section{Sobre este artículo:}

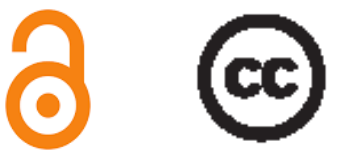

\section{Fuente /s:}

Ana R. C. Donati, Solaiman Shokur, Edgard Morya, Debora S. F. Campos, Renan C. Moioli, Claudia M. Gitti, Patricia B. Augusto, Sandra Tripodi, Cristhiane G. Pires, Gislaine A. Pereira, Fabricio L. Brasil, Simone Gallo, Anthony A. Lin, Angelo K. Takigami, Maria A. Aratanha, Sanjay Joshi, Hannes Bleuler, Gordon Cheng, Alan Rudolph \& Miguel A. L. Nicolelis. Long-Term Training with a BrainMachine Interface-Based Gait Protocol Induces Partial Neurological Recovery in Paraplegic Patients. Scientific Reports. 2016; 6:30383.

Fuente de la Imagen: imagen de NeuroRehabnews.com con fines unicamente ilustrativos.

Autor: Héctor Beltrán Alacreu

Para citar este artículo: Beltrán-Alacreu H. Caminar Después de una Lesión Medular. ¿Ciencia o Milagro? NeuroRehab News 2017 nov; 2 (1): e0028

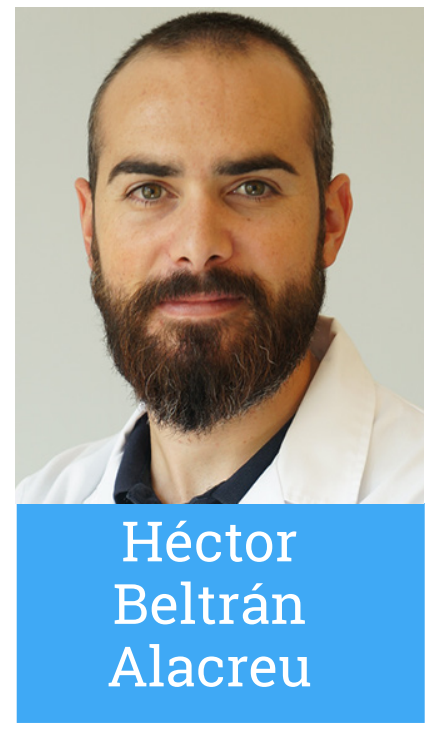

Edición: Alba París Alemany y Juan Manuel García Bechler 